\title{
Cost-effectiveness Analysis of Rituximab Associated With Fludarabine and Cyclophosphamide (R-FC) for the First-line Treatment of Chronic Lymphocytic Leukemia Under the Brazilian Public Health System (SUS).
}

Bruno Azevedo ( $\nabla$ b190960@dac.unicamp.br)

UNICAMP https://orcid.org/0000-0001-6308-2806

Adriana Carvalho

UNICAMP: Universidade Estadual de Campinas

Andre Deeke Sasse

UNICAMP

Research

Keywords: Oncology, leukemia, chronic lymphocytic leukemia, health economic evaluation, costeffectiveness

Posted Date: January 5th, 2021

DOI: https://doi.org/10.21203/rs.3.rs-137750/v1

License: (c) (i) This work is licensed under a Creative Commons Attribution 4.0 International License. Read Full License 


\section{Abstract}

\section{BACKGROUND}

Chronic Lymphocytic leukemia is the most common leukemia in the western world. In the last decade, treatment options expanded with an expressive rise in treatment costs. In Brazil the overall cancer treatment costs are rising mainly due to the judicialization law suits. The efficacy and safety of associating rituximab to fludarabine and cyclophosphamide (R-FC) for the treatment of chronic lymphocytic leukemia has been demonstrated by the CLL8 trial, which was conducted in previously untreated patients positioning the immunochemotherapy regimen as the standard choice for physically fit patients. Considering this scenario, the current study has as objective to evaluate the costeffectiveness of the addition of rituximab to the FC protocol considering the Brazilian public health system perspective.

\section{METHODS}

We developed a Markov model with three health states to extrapolate the CLL8 trial results. The transition probabilities and adverse events incidence were obtained from the trial published data. The costs were assessed from Brazil's ministry of health pricing database (public available). A simplified treatment algorithm was developed according to the current available treatment options in the Brazilian public health system and assuming the availability of rituximab.

\section{RESULTS}

The addition of rituximab to the $\mathrm{FC}$ regimen results in an incremental cost-effectiveness ratio (ICER) of 8389 USD (45003,86 BRL) per year life free of progression. The sensititvity analysis conducted shows a From the Brazilian public health system perspective, the combination of rituximab with the FC chemotherapy represents a cost-effective strategic approach for first-line treatment of patients with CLL.

\section{CONCLUSIONS}

considering the data described in this study, we cqn conclude that the addition of rituximab to the firstline treatment of CLL is cost-effective strategy for the Brazil public health system. With the advent of new drugs and new treatment regimens that presents positive clinical outcomes in patients refratary to the RFC regimen (del17p, p53 mutations), new studies must be conducted to evaluate their cost-effectiveness considering this population of patients.

\section{Introduction}

According to the World Health Organization (WHO) classification of hematopoietic and lymphoid tissues, chronic lymphocytic leukemia (CLL) is a chronic lymphoproliferative disorder characterized by the aberrant proliferation of clonal $\mathrm{CD}^{+} / \mathrm{CD} 23^{+} \mathrm{B}$ cells in the blood, bone marrow and lymphonodes(1). With an estimated incidence of 4.7 new cases per 100.000 individuals, CLL is the most common 
hematopoietic malignancy in the western world(2). The estimated number of new cases in 2020 is 21,040 , making CLL accountable for $1,2 \%$ of all cancer cases and $40 \%$ of all leukemia cases in man and $34 \%$ in woman in USA(3). In Brazil, according with the National Cancer Institute (INCA) data, the estimated number of new cases of leukemia (all types, including CLL) between 2020 and 2023 will be of approximately 11.000 cases (55\% in males and $45 \%$ and females) per year(4).

Compared with more common diseases such as cardiovascular or diabetes, the economic burden of CLL is significantly lower. However, the treatment cost per CLL-case is about twice as high as the common disease patient treatment(5).

Since the publication of the CLL8 trial, the combination of rituximab, fludarabine and cyclophosphamide (R-FC) is the recommend treatment for first-line, fit, CLL patients without comorbidities and normal renal function(6). After this publication, the CLL, and B-cell lymphomas in general, experienced a huge expansion on their therapeutic arsenal. New drugs, including second generation anti-CD20 monoclonal antibodies (ofatumumab and Obinutuzumab), PI3K inhibitors (Idelalisib), Bruton Tyrosine Kinase inhibitors (Ibrutinib) and BCL2 inhibitors (ABT199). These drugs have reduced toxicity in the short term and induce treatment responses, especially in relapse and refractory patients including those with molecular markers associated with poor prognosis such as del17p and p53 mutations.

However, the cost of this target therapies is high, and their utilization must consider the patient physical fitness, del17p deletion, p53 gene mutations, and IGVH mutational status(7). The utilization of this new regimens can potentially bring a significant economic impact for payors and patients. In a populational health economic evaluation, Shanafelt(8) estimates that the utilization of Ibrutininb in the first-line treatment can enhance the CLL treatment costs in approximately $350 \%$. In another report, Chen(9) demonstrates that the cost of CLL treatments overtake that of other cancers.

As the annual cost of cancer care in the USA increased approximately 30\% from 2010 to 2020, the annual cost of CLL treatment is expected to increase by $500 \%$ jumping from 0,7 billion USD in 2011 to 4.2 billion USD in 2020.

Brazil is one of the countries that recognizes a constitutional right to health, and an important part of this right is the access to pharmaceutical drugs. With the approval of new and expensive cancer treatments, the number of lawsuits to secure access to these new drugs increases. According to a report from developed by the Brazilian National Justice Council, the number of lawsuits related to health issues grew $198 \%$ after 2009 to 2017 . From these cases, $69 \%$ were related to the access to new drugs(10) Moreover, a study conducted by Vidal(11) analyzed 158 lawsuits related to antineoplastic drugs, they found that rituximab was present in $23,2 \%$ of the claims analyzed, ranking first in a list that includes antineoplastic drugs for the treatment of all cancer types.

Currently, the Brazilian public health system offers the FC protocol as the only option for CLL first-line treatment for physically fit patients. It is critical for the cost management to have definitions regarding the new available treatments and their indications. Furthermore, to provide health economic evaluation 
data about the cost-effectiveness of the R-FC can provide beneficial outcomes for both patients and public payors in Brazil.

The objective of this study is to evaluate the cost-effectiveness of the rituximab addition to the FC protocol for first line CLL treatment in the Brazilian public health system.

\section{Methods}

\subsection{Model description}

The present study consists of a pharmacoecomomic model that aims to analyze the cost-effectiveness of adding rituximab in combination with the fludarabine plus cyclophosphamide protocol (R-FC) in comparison with the fludarabin plus cyclophosphamide (FC) regimen, as a therapeutic option for CLL naïve treatment patients. A three-state Markov Model was created considering the transition states of first line treatment. The model describes the current clinical practice, and reflects the natural course of the disease according to the treatment option (Fig 1). The model development, costs analysis and other assumptions were done according with the ISPOR consolidated Health Economic Evaluation Reporting Standards (CHEERS)(12)

The model used a cycle length of 3 months and 10 years as time horizon. The Brazilian public healt system willingness to pay is considered as perspective. Future costs and utilities had an annual discount rate of $5 \%$. The Markov Model was developed and calculated with the Tree Age Pro Healthcare ${ }^{\circledR}$ software (Treeage, Williamstown, MA, USA).

All patients started in the "On treatment" state. They could either remain on this state or change to the states "Progression" or "Death". The state "Progression" included all events from first relapse or disease progression after the treatment.

The Cost-effectiveness of R-FC over FC was assessed using an incremental cost-effectiveness ratio (ICER). The transition probabilities were obtained from the CLL8 trial progression-free survival (PFS) and overall survival (OS) curves, up to a follow up period of 3 years. Survival beyond the trial follow up period was projected according to the previous described methodology.

\subsection{Target population}

Patient related data, survival probabilities and clinical outcomes were obtained from the clinical study CLL8 trial (NCT00281918), a global prospective, open label, randomized phase 3 trial(Hallek et al., 2010). The data reported in this publication was used to estimate the long-terms costs relative to the adding of rituximab in the first-line treatment of CLL.

A total of 817 patients diagnosed with CLL and treatment naïve were enrolled and in the trial. The study population had a mean age of 61 years, and $74 \%$ were male. Patients enrolled were in Binet stage $C$ (31\%) or with confirmed active disease in stages A or B (68\%). 
With a median observation time of 3 years after the randomisation, CLL8 trial results demonstrated a significant benefit in R-FC treatment arm related with the progession free-survival (PFS) and overall survival (OS). Table 1 summarizes the clinical data extracted from CLL8.

Table 1

CLL8 clinical data: R-FC versus FC

\begin{tabular}{|ll|}
\hline & $\mathrm{R}-\mathrm{FC}$ versus $\mathrm{FC}$ \\
\hline Progression free survival (PFS) & $\mathrm{HR}=0,56-95 \% \mathrm{Cl}[0,46-0,69]$ \\
\hline Overall survival (OS) & $\mathrm{HR}=0,67-95 \% \mathrm{Cl}[0,48-0,92]$ \\
\hline
\end{tabular}

\subsection{Treatment}

CLL8 study design randomly assigned participants to receive either R-FC or FC treatment regimens as described in the trial protocol. Patients were scheduled to receive 6 cycles of the regimen to which they were randomized. Treatment consisted of six 28 -day intravenous administration of $25 \mathrm{mg} / \mathrm{m}^{2} /$ day of fludarabine and $250 \mathrm{mg} / \mathrm{m}^{2} /$ day of cyclophosphamide on the first 3 days of each cycle. For the R-FC protocol, rituximab was administered intravenously in addition to $\mathrm{FC}$ as $375 \mathrm{mg} / \mathrm{m}^{2}$ before the $\mathrm{FC}$ infusion on day 1 of the first cycle and $500 \mathrm{mg} / \mathrm{m}^{2}$ on day 1 of cycles 2-6.

\subsection{Treatment algorithm}

Although several therapeutic options are currently available for the treatment of first line, treatment naïve CLL patients, there is no formal treatment consensus on the Brazil public health system. An algorithm for the treatment of CLL was developed on the basis of information available in the following guidelines: the International Workshop on CLL (iWCLL) 2018(7), the European Society for Medical Oncology (ESMO)(13), US National Comprehensive Cancer Network (NCCN), clinical guidelines in Oncology: CLL/Small Lymphocytic Lymphoma(14), Brazilian Group of CLL(15).

Using these sources, a preliminary treatment algorithm was developed, this process was them submitted to the review of hematologists with extensive practice in treating CLL in Brazil. The final algorithm that closely reflects the treatment of CLL considering the Brazilian public health experience and the predicted use of rituximab is shown in Fig 2.

\subsection{Costs}

The incremental costs were calculated considering only costs driven by the difference in the treatment arms, the association of rituximab to the FC protocol. The direct costs include drug costs from both protocols, adverse event costs, drug administration costs, and costs with follow-up visits, and laboratory 
tests (clinical examinations, blood exams. Other costs like administrative costs were not included in the model because available study data for these parameters is scarce and they have low representativity.

The assessment of drug prices and costs was obtained from the Brazilian Health Ministry website (SIGTAP - Health procedures and drugs management system), which provides information related to the value paid by the Brazilian Public Health system (SUS) for procedures and medications. Specifically, for CLL there are 2 procedures available for first and second line treatment (nominated APAC's). They were used as main reference for the comparison of incremental costs and the current value allocated for first line CLL treatment.

\subsection{Treatment costs}

The model followed the protocol described on CLL8 trial and summarized briefly in the treatment section. The body surface $\left(\mathrm{m}^{2}\right)$ considered was 1.8 according to the Brazilian populational average of height and weight, according with the Brazilian Institute of Demographics and Statistics (IBGE).

The costs associated with salvage treatment were calculated based on the available guidelines and literature. The treatment costs associated with the R-FC protocol and FC were obtained in Brazilian Reais (BRL) and converted to US Dolars (USD), using a currency exchange rate of 5,39 BRL to 1 USD (currency exchange rates from November $\left.9^{\text {th }} 2020\right)$.

\subsection{Adverse events costs}

To define the costs of adverse events associated with the R-FC protocol, we considered the incidence of adverse events published in the CLL8 trial. The incidence of events reported was significant higher on patients treated with R-FC compared with FC. Among the events reported, grade 3 or 4 adverse events were significant higher in patients treated with rituximab. According to the study protocol, granulocytestimulating factor (G-CSF), was administered to patients that presented this adverse event.

Hospitalizations costs related to adverse events were disregarded as the treatment of neutropenia usually does not require hospital admission.

\subsection{Sensitivity analysis}

Probabilistic sensitivity analysis were performed to determine the magnitude of change in the ICER associated with a range of variations in four most critical parameters. We carried out tornado diagrams to test the model uncertainty when applying variations on the overall survival hazard ratio, the rituximab cost per milligram, the progression-free survival hazard ratio, and 3-month toxicity costs.

\section{Results}

Confirming the clinical findings published on the CLL8 trial and CLL8-follow up $(6,16)$, the main benefit of adding rituximab in the economic model is the lower rate of transitioning between the Markov states. Patients treated with the R-FC protocol spend longer in the unprogressed health state, in a clinical 
remission with non-detected or minimum detectable disease and a relatively good quality of life. The comparison between R-FC and FC resulted in an incremental cost of 6911 USD (37076,14 BRL) with an incremental cost-effectiveness ratio (ICER) of 8389 USD (45003,86 BRL) per year life free of progression.

Rituximab was associated with additional costs including adverse events incidence. The cost related to acquisition of the monoclonal antibody alone accounts for $93 \%$ of the total cost difference between the regimens (see table 2).

Table 2

$\mathrm{R}-\mathrm{FC}$ and FC treatment costs

\begin{tabular}{|llllll|}
\hline & R-FC & \multicolumn{4}{l|}{ FC } \\
& R & F & C & F & C \\
\hline Unit costs* & 608,86 & 42,10 & 2,17 & 42,10 & 2,17 \\
\hline Cost/mg & 1,21 & 0,85 & 0,01 & 0,85 & 0,01 \\
\hline Total drug costs** & 5913,88 & 644,4 & 85,2 & 644,4 & 85,2 \\
\hline Total regimen costs & 6643,48 & & & 729,2 & \\
\hline
\end{tabular}

*Unity drug value obtained from the Health Drug Price database (available at: https://www.saude.gov.br)

**Drug costs calculate having the CLL8 protocol as reference for FC and R-FC regimen. 6 cycles of Fludarabine $25 \mathrm{mg} / \mathrm{m}^{2}$ and cyclophosphamide $250 \mathrm{mg} / \mathrm{m}^{2}$. The analysis considers $1,7 \mathrm{~m}^{2}$ as an average value for Brazilian patients.

Values are displayed in USD (5,39 BRL to 1 USD exchange rate)

Salvage therapy accounted for significant costs in each treatment arm. Costs associated with salvage therapy were similar in both arms (table 3). Nevertheless, these costs incurred at an earlier time point in $\mathrm{FC}$ arm when compared with R-FC treatment, yielding an incremental cost in the FC arm.

Table 3

Salvage treatment costs

\begin{tabular}{|llll|}
\hline & V & C & P \\
\hline Unit costs* & 3,71 & 2,17 & 0,01 \\
\hline Cost/mg & 3,71 & 0,01 & 0,01 \\
\hline Total drug costs & 59,36 & 102 & 0,82 \\
\hline Total regimen costs & 162,18 & & \\
\hline
\end{tabular}

*Unity drug value obtained from the Health Drug Price database (available at: https://www.saude.gov.br) 
The analysis considers $1,7 \mathrm{~m}^{2}$ as an average value for Brazilian patients.

Values are displayed in USD (5,39 BRL to 1 USD exchange rate)

VCP protocol according with Marcus et al(17).

\subsection{Sensitivity analysis}

In general, the results were shown to be robust in the sensitivity analyses. Toxicity costs, PFS HR, overall survival HR, and rituximab cost were the drivers for cost-effectiveness of the monoclonal antibody. The analysis outcomes points to a model sensitivity related to the overall survival hazard ratio alterations. The tornado diagram (fig 3) displays the results found.

\section{Discussion}

This study evaluates the cost-effectiveness of the R-FC regimen for the first line treatment of CLL patients versus the FC protocol, currently available for this segment of patients in the Brazilian public health system. The modeled population was based on the patients enrolled in the CLL8 trial. The analysis confirmed that the addition of rituximab results in a significant improvement in health gains with moderate additional costs.

Despite the structural differences between distinct country's health systems, the results of this study endorse other published economic analysis from Australia(18), Spain(19), and two other health economic evaluations $(5,20)$ with the same objective. In these health evaluation studies, adding rituximab has been shown to be cost-effective with ICERs between 19000 USD - 28500 USD. With an ICER of 8388,73 USD, the present analysis shows a considerable decrease in the incremental cost-effectiveness ratio. Assuming the decrease in rituximab prices since the publication of the studies and, also, by the fact that we used the biosimilar anti-CD20 prices, it is possible to justify the scenario, which is very favorable to a potential adoption of the immunochemotherapy regimen for this segment of patients. Because the drug costs for rituximab represents a major proportion $(>90 \%)$ of the incremental costs for the R-FC regimen, the unity cost of the drug have a significant impact on the cost-effectiveness analysis, thus, governmental initiatives that could potentially reduce the price, will yield a positive economic outcome.

According with the WHO Commission on Macroeconomics and Health, in developing countries with no definition on the cost-effectiveness threshold, health interventions can be considered as cost-effective when the ICER value is between 1 to 3 times the country's per capita GDP (gross domestic product). According with the IBGE, Brazil's per capita GDP was 5667 USD (30407 BRL) in 2016.

In conclusion, considering the data described in this study, we cqn conclude that the addition of rituximab to the first-line treatment of CLL is cost-effective strategy for the Brazil public health system. With the advent of new drugs and new treatment regimens that presents positive clinical outcomes in patients 
refratary to the R-FC regimen (del17p, p53 mutations), new studies must be conducted to evaluate their cost-effectiveness considering this population of patients.

\section{Declarations}

1 ETHICS APPROVAL

The current study does not demand ethics approval.

2 CONSENT FOR PUBLICATION

Not applicable

3 AVAILABILITY OF DATA AND MATERIAL

The datasets generated during and/or analysed during the current study are available in the following weblinks:

- Costs analysis: SIGTAP table: www.sigtap.datasus.gov.br

- CLL8 Trial: Lancet 2010 Oct 2;376(9747):1164-74. doi: 10.1016/S0140-6736(10)61381-5.

4 COMPETING INTERESTS

The authors declare that they have no competing interests

5 FUNDING

Not applicable. This research has not received any financial support.

6 ACKNOWLEDGEMENTS

Not applicable.

\section{Bibliography}

1. SH S, E C, SA P, NL H, H S, R S, et al. The 2016 revision of the World Health Organization classification of lymphoid neoplasms. Blood. 2016;127(20).

2. F B, R D-F. Chronic lymphocytic leukaemia: from genetics to treatment. Nature reviews Clinical oncology. 2019;16(11).

3. RL S, KD M, A J. Cancer statistics, 2020. CA: a cancer journal for clinicians. 2020;70(1).

4. Estimativa 2020 : incidência de câncer no Brasil / Instituto Nacional de Câncer José Alencar Gomes da Silva. Rio de Janeiro: INCA; 2019. p. 122. 
5. D M, K F, P K, B E, R W, M R, et al. Cost-effectiveness of rituximab in addition to fludarabine and cyclophosphamide (R-FC) for the first-line treatment of chronic lymphocytic leukemia. Leukemia \& lymphoma. 2016;57(5).

6. M H, K F, G F-R, AM F, R B, J M, et al. Addition of rituximab to fludarabine and cyclophosphamide in patients with chronic lymphocytic leukaemia: a randomised, open-label, phase 3 trial. Lancet (London, England). 2010;376(9747).

7. $M H, B D C, D C, F C-C, G D, H D$, et al. iwCLL guidelines for diagnosis, indications for treatment, response assessment, and supportive management of CLL. Blood. 2018;131(25).

8. TD S, BJ B, HD F, KG C, W D, JF L, et al. Impact of ibrutinib and idelalisib on the pharmaceutical cost of treating chronic lymphocytic leukemia at the individual and societal levels. Journal of oncology practice. 2015;11(3).

9. Q C, N J, T A, WG W, CR F, SM OB, et al. Economic Burden of Chronic Lymphocytic Leukemia in the Era of Oral Targeted Therapies in the United States. Journal of clinical oncology : official journal of the American Society of Clinical Oncology. 2017;35(2).

10. Council BNJ. Judicialização da saúde no Brasil: perfil das demandas, causas e propostas de solução. São Paulo: Brazil National Justice Council; 2019.

11. TJ V, EL M, MPF R, MJSD S. The lawsuits to antineoplastic drugs: the tip of an iceberg? Ciencia \& saude coletiva. 2017;22(8).

12. D H, M D, S P, C C, D M, D G, et al. Consolidated Health Economic Evaluation Reporting Standards (CHEERS)-explanation and elaboration: a report of the ISPOR Health Economic Evaluation Publication Guidelines Good Reporting Practices Task Force. Value in health : the journal of the International Society for Pharmacoeconomics and Outcomes Research. 2013;16(2).

13. B E, T R, E M, P G, CU N, AP K, et al. Chronic lymphocytic leukaemia: ESMO Clinical Practice Guidelines for diagnosis, treatment and follow-up †. Annals of oncology : official journal of the European Society for Medical Oncology. 2020.

14. WG W, AD Z, LI G, JS A, RH A, CB A, et al. NCCN Guidelines Insights: Chronic Lymphocytic Leukemia/Small Lymphocytic Lymphoma, Version 1.2017. Journal of the National Comprehensive Cancer Network: JNCCN. 2017;15(3).

15. CA R, MV G, MR I, I L-M, AD P, DL F, et al. Diagnosis and treatment of chronic lymphocytic leukemia: recommendations from the Brazilian Group of Chronic Lymphocytic Leukemia. Revista brasileira de hematologia e hemoterapia. 2016;38(4).

16. K F, J B, AM F, V G, CD H, P C, et al. Long-term remissions after FCR chemoimmunotherapy in previously untreated patients with CLL: updated results of the CLL8 trial. Blood. 2016;127(2).

17. R M, K I, A B, D C, E F, J C, et al. CVP chemotherapy plus rituximab compared with CVP as first-line treatment for advanced follicular lymphoma. Blood. 2005;105(4).

18. M A, J H, SP M, C T, G M. Modelling the cost effectiveness of rituximab in chronic lymphocytic leukaemia in first-line therapy and following relapse. PharmacoEconomics. 2014;32(2). 
19. LF C, JA GM, F G, M G, E R, J dIS, et al. [Economic evaluation of rituximab added to fludarabine plus cyclophosphamide versus fludarabine plus cyclophosphamide for the treatment of chronic lymphocytic leukemia]. Gaceta sanitaria. 2011;25(4).

20. J H, C R, A S, S L, M F, L H, et al. Cost-effectiveness of adding rituximab to fludarabine and cyclophosphamide for the treatment of previously untreated chronic lymphocytic leukemia. Leukemia \& lymphoma. 2012;53(2).

\section{Figures}

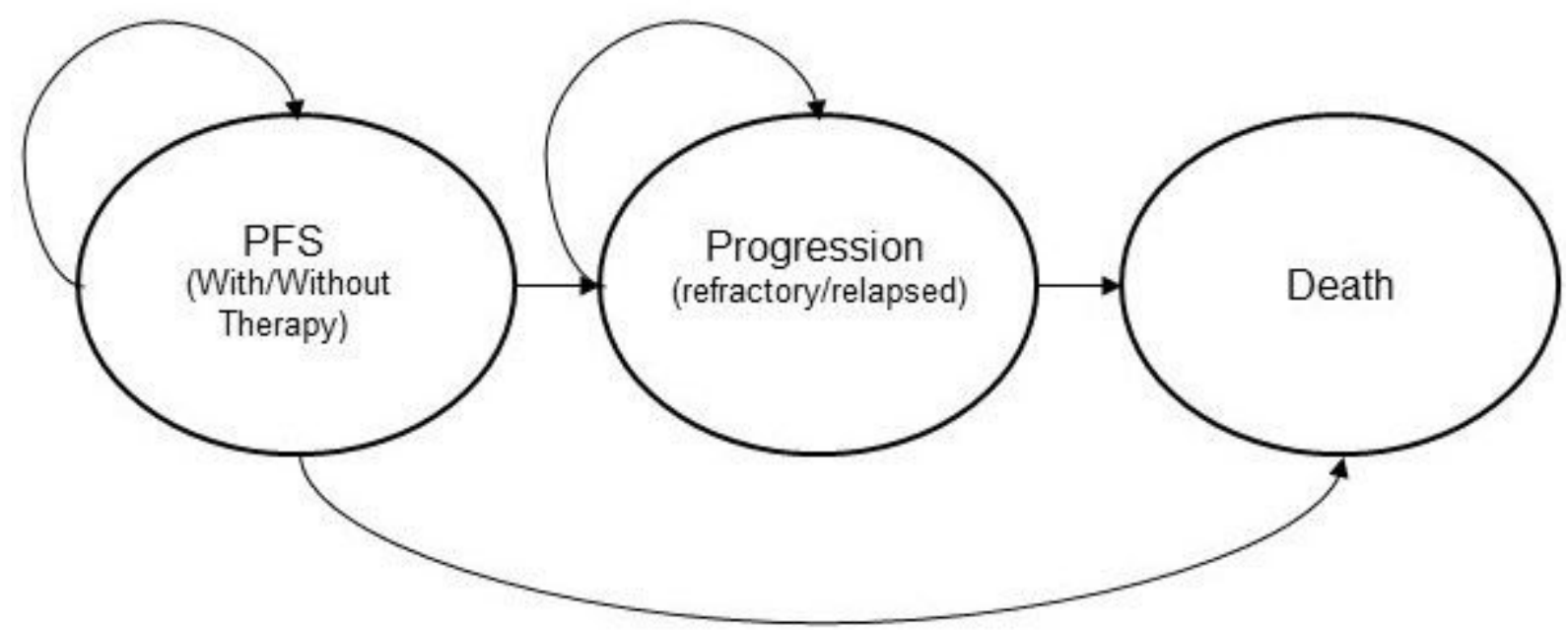

Figure 1

Markov Model structure. Three mutually exclusive health states were included: "PFS (with or without therapy)", "progression", and "death". The possible transitions between the health states are indicated by the arrows. Patients start in the first health state and can move to another one or stay in the same state at the end of each cycle analysis. 


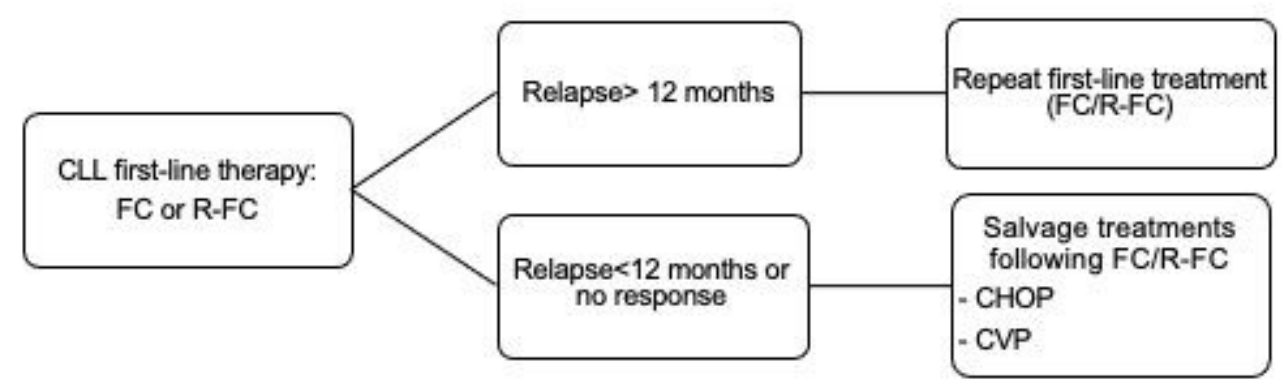

Figure 2

Simplified CLL treatment algorithm assuming rituximab availability

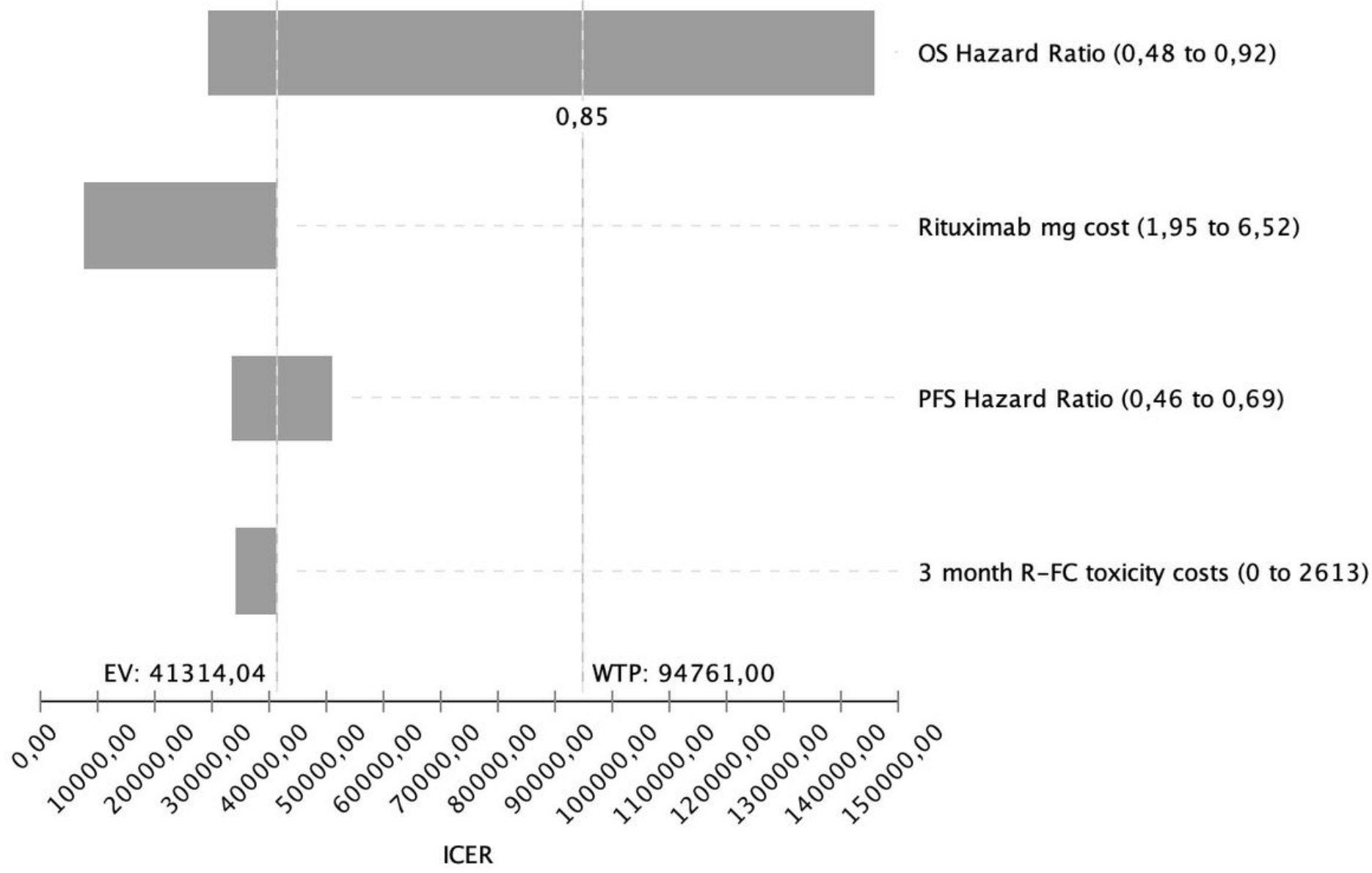


Figure 3

Tornado diagram. The graphic displays the sensitivity of the model to four selected parameters.

\section{Supplementary Files}

This is a list of supplementary files associated with this preprint. Click to download.

- CHEERSChecklist.pdf 